\title{
On a Large Sample Test for the Traffic Intensity in $G I|G| s$ Queue
}

\author{
S. Subba Rao \\ Washington State University, Pullman, Washington, USA \\ K. Harishchandra \\ Bangalore University, Bangalore, India
}

\begin{abstract}
A large sample test based on normal approximation for the traffic intensity parameter $\rho$ in the cases of single and multiple-server queues has been proposed. The test procedure is developed without imposing steady-state assumptions and is applicable to queueing systems with general interarrival and service-time distributions.
\end{abstract}

\section{INTRODUCTION}

Statistical inference, both estimation and hypothesis testing, plays a vital role in the analysis of queues, particularly when a practical congestion problem is to be tackled by empirical methods. Some of the literature on inference as applied to queues can be found in Harris [8], Bhat and Subba Rao [4], and Basawa and Prabhu [1]. Clarke [5], Benẽs [2], Cox [6], and Basawa and Prabhu [1] have investigated the problem of parameter estimation in Poisson/exponential and birth-death type queuing models. Cox [6], Wolff [12], and Thiagarajan and Harris [11] have discussed likelihood ratio tests and a few large sample tests for the queueing parameters such as arrival/service rates of $M|M| 1$ and other birth-death type queues.

The traffic intensity parameter $\rho$ is the most important system parameter, where $\rho$ is the ratio of mean service time to mean interarrival time. Since an infinite combinations of these two parameters leads to a single value of $\rho$, from the point of view of design and control of queues it is desirable to have test procedures for $\rho$ alone. Some of the reported work in the literature regarding hypothesis testing concerning $\rho$ includes the $F$ test in the case of $M|M| 1$ queue due to Cox [6], a control chart technique proposed by Bhat and Subba Rao [3], and a sequential probability ratio test for $\rho$ in $M|G| 1$ and $G I|M| s$ queues due to Subba Rao et al. [10].

In this paper we propose a large sample test for $\rho$ based on normal approximations. The procedure is applicable in the case of single and multiple-server queues with general input and service-time distributions. No steady-state assumptions are imposed while deriving the test procedures.

\section{THE GI $|G| 1$ QUEUE}

Consider a single-server queue. The interarrival times are assumed to be independent identically distributed (i.i.d) random variables with distribution function (d.f.) $F(x)$ with mean $\mu_{x}$ and variance $\sigma_{x}^{2}$. The service times are also assumed to be i.i.d random 
variables with d.f. $G(y)$ and with mean $\mu_{y}$ and variance $\sigma_{y}^{2} . \sigma_{x}^{2}$ and $\sigma_{y}^{2}$ are assumed to be finite. Then the traffic intensity parameter $\rho=\mu_{y} / \mu_{x}$.

Assume that the first customer arrives at time $t=0$. For the purpose of testing we observe the system until the first $n$ customers leave the system. We denote by $\tau_{n}$ the $n$th departure epoch. The service times of these $n$ customers are denoted by $y_{1}, y_{2}, \ldots, y_{n}$.

We shall denote the successive interarrival times by $x_{1}, x_{2}, \ldots$, etc. The interarrival times of all the customers who arrive during $\left(0, \tau_{n}\right]$ are observed. Thus we get the observations $x_{1}, x_{2}, \ldots, x_{m}$ where

$$
m=\operatorname{Max}\left\{j: x_{1}+x_{2}+\cdots+x_{j} \leq \tau_{n}\right\} .
$$

Note that $m \geq n$.

$$
\text { Let } \bar{x}_{m}=\sum_{1}^{m} x_{i} / m, \quad \bar{x}_{n}^{*}=\sum_{1}^{n} x_{i} / n, \quad \bar{y}_{n}=\sum_{1}^{n} y_{j} / n .
$$

It may be observed that $\bar{x}_{n}^{*}$ and $\bar{y}_{n}$ are independent, but $\bar{x}_{m}$ and $\bar{y}_{n}$ are not, due to (1). Basawa and Prabhu [1] have shown that the asymptotic behavior of $\bar{x}_{m}$ and $\bar{x}_{n}^{*}$ are similar, namely, both are consistent estimates of $\mu_{x}$ and asymptotically normal with $\bar{x}_{m}$ having a smaller variance. Further $\bar{x}_{m}$ and $\bar{y}_{n}$ are asymptotically independent.

We shall state below a few results which have been proved by Basawa and Prabhu [1], relating to the sequences $\left\{x_{n}\right\}$ and $\left\{y_{n}\right\}$.

$$
\begin{gathered}
\frac{m}{n} \stackrel{P}{\rightarrow} \beta, \quad \text { where } \beta=\max (1, \rho), \\
\bar{x}_{m} \stackrel{a \cdot s}{\rightarrow} \mu_{x}, \quad \bar{x}_{n}^{*} \stackrel{a \cdot s}{\rightarrow} \mu_{x}, \quad \bar{y}_{n} \stackrel{a \cdot s}{\rightarrow} \mu_{y}, \\
\sqrt{n}\left(\bar{x}_{n}^{*}-\mu_{x}\right) \stackrel{L}{\rightarrow} N\left(0, \sigma_{x}^{2}\right), \\
\sqrt{m}\left(\bar{x}_{m}-\mu_{x}\right) \stackrel{L}{\rightarrow} N\left(0, \sigma_{x}^{2} / \beta\right), \\
\sqrt{n}\left(\bar{y}_{n}-\mu_{y}\right) \stackrel{L}{\rightarrow} N\left(0, \sigma_{y}^{2}\right), \\
\bar{x}_{m} \text { and } \bar{y}_{n} \text { are asymptotically independent. }
\end{gathered}
$$

By the strong law of large numbers it follows that $\bar{x}_{n}^{*}$ and $\bar{y}_{n}$ converge to their respective means with probability one. Since $m \uparrow \infty$ as $n \rightarrow \infty$ and $m / n \rightarrow \beta$ (a constant), it may be seen that $\bar{x}_{m}$ also converges to $\mu_{x}$ with probability one. Results (4) and (6) follow from the central limit theorem. The results (5) and (7), namely, $\bar{x}_{m}$ is asymptotically normal and $\bar{x}_{m}$ and $\bar{y}_{n}$ are asymptotically independent, have been proved by the authors [1] by using the central limit theorem, showing that the asymptotic joint distribution of $\bar{x}_{m}$ and $\bar{y}_{n}$ is a bivariate normal distribution with covariance term zero. Due to the characterization property of normal distribution it follows that $\bar{x}_{m}$ and $\bar{y}_{n}$ are asymptotically independent and follow normal distribution. No steady-state assumptions are imposed on the system while deriving the above results.

Since $\bar{x}_{m}$ has a smaller variance as compared to $\bar{x}_{n}^{*}$ and due to (7), we shall use $\bar{x}_{m}$ as an estimate of $\mu_{x}$, while deriving the large sample tests for $\rho$.

We state our hypothesis as $H: \rho=\rho_{0}$ to be tested against $K: \rho \neq \rho_{0}$.

Let $\phi(t)=\log t$. Define $\delta=\phi\left(\mu_{y}\right)-\phi\left(\mu_{x}\right)$, then $\delta=\log \mu_{y}-\log \mu_{x}=\log \rho$, since $\rho=\mu_{y} / \mu_{x}$. Then the hypothesis $H: \rho=\rho_{0}$ vs $K: \rho \neq \rho_{0}$ reduces to $H^{\prime}: \delta=\delta_{0}$ vs $K^{\prime}: \delta \neq \delta_{0}$, where $\delta_{0}=\log \rho_{0}$.

Based on the estimates $\bar{x}_{m}$ and $\bar{y}_{n}$ discussed previously, define $\delta_{m n}=\phi\left(\bar{y}_{n}\right)-$ 
$\phi\left(\bar{x}_{m}\right)$. Since, from (5) and (6),

$$
\sqrt{m}\left(\bar{x}_{m}-\mu_{x}\right) \stackrel{L}{\rightarrow} N\left(0, \sigma_{x}^{2} / \beta\right)
$$

and

$$
\sqrt{n}\left(\bar{y}_{n}-\mu_{y}\right) \stackrel{L}{\rightarrow} N\left(0, \sigma_{y}^{2}\right),
$$

it follows from Lemma 1, Eq. (12) (given at the end of the paper) that

$$
\sqrt{m}\left[\phi\left(\bar{x}_{m}\right)-\phi\left(\mu_{x}\right)\right] \stackrel{L}{\rightarrow} N\left(0, \sigma_{x}^{2} / \beta \mu_{x}^{2}\right)
$$

and

$$
\sqrt{n}\left[\phi\left(\bar{y}_{n}\right)-\phi\left(\mu_{y}\right)\right] \stackrel{L}{\rightarrow} N\left(0, \sigma_{y}^{2} / \mu_{y}^{2}\right)
$$

Since $\bar{x}_{m}$ and $\bar{y}_{n}$ are asymptotically independent, $\phi\left(\bar{x}_{m}\right)$ and $\phi\left(\bar{y}_{n}\right)$ are also. Hence, from Eqs. (8) and (9) using Lemma 2, Eq. (14), it follows that the statistic

$$
T_{m n}=\frac{m n \beta \delta_{m n}}{m \beta \eta_{y}^{2}+n \eta_{x}^{2}}
$$

has a normal distribution with mean $\left(m n \beta \delta / m \beta \eta_{y}^{2}+n \eta_{x}^{2}\right)$ and variance $\left(m n \beta / m \beta \eta_{y}^{2}+n \eta_{x}^{2}\right)$ for large $m$ and $n$, where

$$
\eta_{x}=\sigma_{x} \phi^{\prime}\left(\mu_{x}\right)=\sigma_{x} / \mu_{x}
$$

and

$$
\eta_{y}=\sigma_{y} \phi^{\prime}\left(\mu_{y}\right)=\sigma_{y} / \mu_{y}
$$

Therefore

$$
Z=\left(\frac{m n \beta}{m \beta \eta_{y}^{2}+n \eta_{x}^{2}}\right)^{1 / 2}\left(\delta_{m n}-\delta\right)
$$

has an asymptotic normal distribution with mean 0 and variance 1 .

Thus, for testing $H: \rho=\rho_{0}$ vs $K: \rho \neq \rho_{0}$ in terms of $H^{\prime}: \delta=\delta_{0}$ vs $K^{\prime}: \delta \neq \delta_{0}$, the statistic $Z$ given by (11) can be made use of.

Since the unknown parameters $\beta, \eta_{x}=\sigma_{x} / \mu_{x}$, and $\eta_{y}=\sigma_{y} / \mu_{y}$ appear in the statistic $Z$, we have a problem. However, $\beta$ can be replaced by its consistent estimate $\hat{\beta}=$ $m / n$ [from Eq. (2)] and due to Lemma 1, Eq. (13), the normal approximation still holds.

Interestingly, the quantities $\eta_{x}$ and $\eta_{y}$ turn out to be constants for exponential and Erlangian distributions because $\phi(t)=\log t$ is the variance stabilizing transformation for these distributions. Hence in these cases the problem is automatically solved.

It may be observed that $\eta_{x}$ and $\eta_{y}$ are the coefficients of variation for the populations of $X$ and $Y$, respectively. Therefore, if we have prior knowledge about these coefficients of variation, they can be used in the statistic $Z$ in the cases of non-Erlangian distributions.

In the absence of any knowledge about $\eta_{x}$ and $\eta_{y}$, they can be replaced by their respective estimates, namely, $\hat{\eta}_{x}=S_{x} / \bar{x}_{m}$ and $\hat{\eta}_{y}=S_{y} / \bar{y}_{n}$, where

$$
S_{x}^{2}=\sum_{i} \frac{\left(x_{i}-\bar{x}_{m}\right)^{2}}{m}
$$


and

$$
S_{y}^{2}=\sum_{j} \frac{\left(y_{j}-\bar{y}_{n}\right)^{2}}{n}
$$

Then from Lemma 2, Eq. (15), it follows that $\hat{\eta}_{x}$ and $\hat{\eta}_{y}$ are consistent estimates of $\eta_{x}$ and $\eta_{y}$, respectively. Hence from Eq. (13) of Lemma 1 ,

$$
Z^{\prime}=\left(\frac{m n \hat{\beta}}{m \hat{\beta} \hat{\eta}_{y}^{2}+n \hat{\eta}_{x}^{2}}\right)^{1 / 2}\left(\delta_{m n}-\delta\right)
$$

follows a normal distribution with mean 0 and variance 1 for large $m$ and $n$.

EXAMPLE 1: THE $M|M| 1$ QUEUE. Here we have $F(x)=1-e^{-\lambda x}, G(y)=$ $1-e^{-\mu y}$ so that $\mu_{x}=1 / \lambda, \mu_{y}=1 / \mu, \rho=\lambda / \mu, \sigma_{x}^{2}=1 / \lambda^{2}$, and $\sigma_{y}^{2}=1 / \mu^{2}$. Hence $\eta_{x}=\sigma_{x} \phi^{\prime}\left(\mu_{x}\right)=1, \eta_{y}=\sigma_{y} \phi^{\prime}\left(\mu_{y}\right)=1$. We shall take $\hat{\beta}=m / n$. Then we have

$$
Z=\left(\frac{m^{2} n}{m^{2}+n^{2}}\right)^{1 / 2}\left(\delta_{m n}-\delta\right) \sim N(0,1) .
$$

EXAMPLE 2: THE $E_{k}\left|E_{l}\right| 1$ QUEUE. In this case

$$
\begin{aligned}
F(x) & =\int_{0}^{x} \frac{(k \lambda)^{k}}{\sqrt{(k)}} e^{-k \lambda u} u^{k-1} d u, \\
G(y) & =\int_{0}^{y} \frac{(l \mu)^{l}}{\sqrt{(l)}} e^{-l \mu u} u^{l-1} d u, \\
\mu_{x}=1 / \lambda, \quad \mu_{y} & =1 / \mu, \quad \sigma_{x}^{2}=1 / k \lambda^{2}, \quad \sigma_{y}^{2}=1 / l \mu^{2},
\end{aligned}
$$

and hence $\eta_{x}=1 / \sqrt{k}$ and $\eta_{y}=1 / \sqrt{l}$. As in the previous case we shall take $\hat{\beta}=$ $m / n$. On substitution in (11) the statistic $Z$ reduces to

$$
Z=\left(\frac{m^{2} n l k}{m^{2} k+n^{2} l}\right)^{1 / 2}\left(\delta_{m n}-\delta\right) \sim N(0,1)
$$

\section{THE $G I|G| s$ QUEUE}

As in the $G I|G| 1$ queue the d.f. of the interarrival time is denoted by $F(x)$ with mean $\mu_{x}$ and variance $\sigma_{x}^{2}$. We assume that there are $s$ servers in the system working in parallel independently of each other. The service time has a common d.f. $G(y)$ for each server with mean $\mu_{y}$ and variance $\sigma_{y}^{2}$.

The system is observed until the $n$th departure. The service times are denoted by $y_{1}, y_{2}, \ldots, y_{n}$. The interarrival times of all the customers who arrive before the $n$th departure are observed. We shall denote these observations by $x_{1}, x_{2}, \ldots, x_{m}$.

In this case $\rho=\mu_{y} / s \mu_{x}$. Let $\phi(t)=\log t$. Define $\delta=\phi\left(\mu_{y}\right)-\phi\left(s \mu_{x}\right)=\log \mu_{y} /$ $s \mu_{x}=\log \rho$. Thus, testing $H: \rho=\rho_{0}$ vs $K: \rho \neq \rho_{0}$ is equivalent to testing $H^{\prime}: \delta=\delta_{0}$ vs $K^{\prime}: \delta \neq \delta_{0}$. Define $\delta_{m n}=\phi\left(\bar{y}_{n}\right)-\phi\left(s \bar{x}_{m}\right)$.

On the similar lines of arguments described in Section 2, it can be shown that the statistic

$$
T_{m n}=\frac{m n \beta \delta_{m n}}{m \beta \eta_{y}^{2}+n s^{2} \eta_{x}^{2}}
$$


has a normal distribution with mean $\left(m n \beta \delta / m \beta \eta_{y}^{2}+n s^{2} \eta_{x}^{2}\right)$ and variance $\left(m n \beta / m \beta \eta_{y}^{2}+n s^{2} \eta_{x}^{2}\right)$ for large $m$ and $n$, so that the statistic

$$
Z=\left(\frac{m n \beta}{m \beta \eta_{y}^{2}+n s^{2} \eta_{x}^{2}}\right)^{1 / 2}\left(\delta_{m n}-\delta\right) \sim N(0,1)
$$

For the $E_{k}\left|E_{l}\right| s$ system the statistic $Z$ reduces to

$$
Z=\left(\frac{m n l k \beta}{m k \beta+n s^{2} l}\right)^{1 / 2}\left(\delta_{m n}-\delta\right) .
$$

For other multiserver queues, $\eta_{x}^{2}$ and $\eta_{y}^{2}$ are replaced by their respective consistent estimates.

Certain results obtained in the earlier discussions follow from the following lemmas. For the proofs and detailed discussions see Rao [9, p. 385] and Cramér [7, p. 254].

LEMMA 1: Let $\left\{T_{n}\right\}, n=1,2, \ldots$ be a sequence of statistics such that $\sqrt{n}\left(T_{n}-\theta\right) \stackrel{L}{\rightarrow} N\left[0, \sigma^{2}(\theta)\right]$, where $s \cdot e\left(T_{n}\right)=\sigma(\theta) / \sqrt{n}$.

Let $\phi$ be a function of a single variable admitting the first derivative $\phi^{\prime}$, then

$$
\sqrt{n}\left[\phi\left(T_{n}\right)-\phi(\theta)\right] \stackrel{L}{\rightarrow} N\left[0,\left\{\phi^{\prime}(\theta) \sigma(\theta)\right\}^{2}\right] .
$$

Further if $\phi^{\prime}(\theta) \neq 0, \phi^{\prime}(\theta)$, and $\sigma(\theta)$ are continuous, then

$$
\frac{\sqrt{n}\left[\phi\left(T_{n}\right)-\phi(\theta)\right]}{\phi^{\prime}\left(T_{n}\right) \sigma\left(T_{n}\right)} \stackrel{\llcorner}{\rightarrow} N(0,1) .
$$

LEMMA 2: Let $\left\{X_{n}\right\}$ and $\left\{Y_{n}\right\}, n=1,2, \ldots$ be sequences of random variables, then

$$
\begin{aligned}
& \text { If } X_{n} \stackrel{P}{\rightarrow} X \text { and } Y_{n} \stackrel{P}{\rightarrow} Y \text {, then } X_{n} \pm Y_{n} \stackrel{P}{\rightarrow} X \pm Y \text {. } \\
& \text { If } X_{n} \stackrel{P}{\rightarrow} a \text { and } Y_{n} \stackrel{P}{\rightarrow} b(a, b \text { are constants and } b \neq 0) \text {, } \\
& \text { then } \frac{X_{n}}{Y_{n}} \stackrel{P}{\rightarrow} \frac{a}{b} .
\end{aligned}
$$

\section{REMARKS}

The proposed large sample test for the traffic intensity parameter, unlike, the $F$ test due to Cox [6] for the $M|M| 1$ queue and the sequential probability ratio test due to Subba Rao and others [10] for the $M|G| 1$ and $G I|M| s$ queues, is a simple test procedure which is applicable to single and multiserver queues with general input and service time distributions. Further, it was observed that for Erlangian type input/output distributions the test statistic is a function of $\rho$ alone and does not require estimates of the population parameters. The efficiency of the proposed test procedures depends on our basic assumptions on normal approximations and asymptotic independence. Since these results have been deduced from the central limit theorem, normality and hence independence is approached rapidly even for relatively small sample sizes. Hence the test procedures are expected to be powerful for even moderate sample sizes. 


\section{ACKNOWLEDGMENT}

The authors are grateful to the referee for suggesting some corrections in the first draft of the paper.

\section{REFERENCES}

[1] Basawa, I. V., and Prabhu, N. U., "Estimation in Single Server Queues," Naval Research Logistics Quarterly, 28, 475-487 (1981).

[2] Benēs, V. E., "A Sufficient Set of Statistics for a Simple Telephone Exchange Model," Bell System Journal, 36, 939-964 (1957).

[3] Bhat, U. N., and Subba Rao, S., "A Statistical Technique for the Control of Traffic Intensity in Queueing Systems $M|G| 1$ and $G I|M| 1$," Operations Research, 20, 955-966 (1972).

[4] Bhat, U. N., and Subba Rao, S., "'Statistical Analysis of Queueing Systems,"' Proceedings of the American Statistical Association, Houston meeting, 36-45 (1980).

[5] Clarke, A. B., "Maximum Likelihood Estimates in a Simple Queue," Annals of Mathematical Statistics, 28, 1036-1040 (1957).

[6] Cox, D. R., "Some Problems of Statistical Analysis with Congestion," Proceedings of the Symposium on Congestion Theory, W. L. Smith and W. E. Wilkinson, Eds. University of North Carolina Press, Chapel Hill, NC, 1965.

[7] Cramér, H., Mathematical Methods of Statistics, Princeton Univ. Press, Princeton, NJ, 1962.

[8] Harris, C. M., "Some New Results in the Statistical Analysis of Queues," Proceedings of Conference on Mathematical Methods in Queueing Theory, A. B. Clarke, Ed. SpringerVerlag, New York, 1973, Vol. 98, pp. 157-183.

[9] Rao, C. R., Linear Statistical Inference, 2nd ed., Wiley, New York, 1973.

[10] Subba Rao, S., Bhat, U. N., and Harishchandra, K., "Control of Traffic Intensity in a Queue-A Method Based on SPRT," Opsearch, 21, 63-80 (1984).

[11] Thiagarajan, T. R., and Harris, C. M., "Statistical Tests for Exponential Service from $M|G| 1$ Waiting-Time Data," Naval Research Logistics Quarterly, 26, 511-520 (1979).

[12] Wolff, R. W., "Problems of Statistical Inference for Birth and Death Queueing Models," Operations Research, 13, 343-357 (1965). 